\title{
Welcome Message from TPC Chairs
}

It is my great pleasure to welcome all the participants to the 16th International Symposium on Communications and Information Technologies (ISCIT 2016) as TPC chairs. ISCIT 2016 will provide a forum for researchers, engineers, and industry experts to discuss recent development, new ideas and breakthrough in communications and information technologies.

As focused by the experts and scholars within the field, ISCIT 2016 pay close attention to the emerging new technologies and the existing key technologies. We are honored to have the keynote speakers invited to present their outstanding achievements and understanding on the following topics: optical technologies for the next generation wireless networks, applications and basics of network coding, future generations of wireless networks, telecom operator big data development.

ISCIT 2016 have received 428 papers submitted by authors from 14 countries and regions, and finally, 155 papers are accepted. The accepted papers will be presented and discussed in 14 regular technical sessions, 4 workshops, and 3 special sessions.

To further enable the discussion on the new approaches, the following topics are included in the symposium: communication systems, VLSI and circuits and systems, signal and image processing, computer and information networks, big data research and application, wireless communication research for petroleum industry, space-ground integrative information network, 5G \& beyond - enabling technologies and applications, big data intelligence research for special group, and telecom operation research and optimization.

We would like to express our sincere appreciation to the TPC members and reviewers for their tremendous efforts. Especially, we appreciate all the technical sponsors for their generous support and advice, including IEEE Circuits and Systems Society (CAS), Engineering Sciences Society, the Institute of Electronics, Information and Communication Engineers (IEICE ESS), and Electrical Engineering/Electronics, Computer, Telecommunications and Information Technology Association of Thailand (ECTI). Finally, we would also like to thank all the authors and participants for their excellent work and cooperation

\section{ISCIT 2016 TPC Chairs}

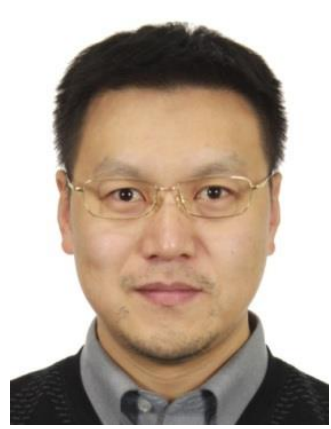

Songlin Sun, Beijing University of

Posts and Telecommunications, China

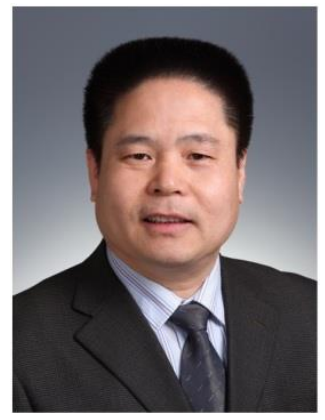

Ju Liu, Shandong University, China

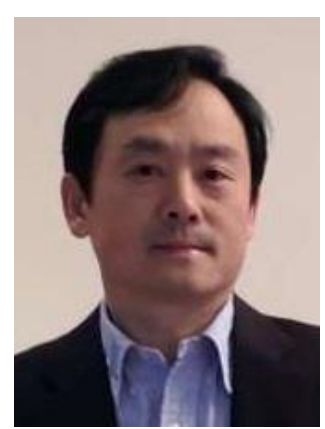

Xiaojing Huang, University of Technology Sydney, Australia

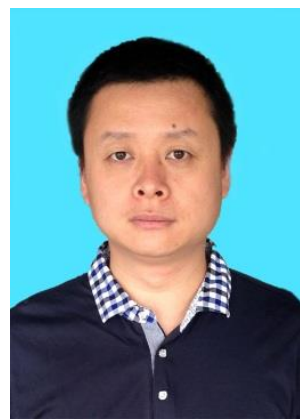

Xinzhou Cheng, China Unicom Network

Technology Research Institute, China 